\title{
The influence of preoperative anemia on 30-day mortality and perioperative myocardial infarction among older patients undergoing non-cardiac surgery: A retrospective cohort study
}

Sim Yilin Eileen, Abdullah Hairil Rizal

Department of Anaesthesia, Singapore General Hospital

$$
\text { Background/Introduction }
$$

Preoperative anemia is common among older patients undergoing major non-cardiac surgeries and is associated with increased risk of postoperative mortality and morbidity. We aim to investigate the relationship between preoperative anaemia with $\mathbf{3 0}$ day mortality rate and perioperative myocardial infarction (PMI) among older patients who underwent non-cardiac surgery. We defined PMI based on the European Perioperative Clinical Outcome consensus statement.

\section{Methodology}

- Retrospective single center cohort study with 24,579 patients above 65 years who underwent index surgery between 1 Jan 2012 - 31 Oct 2016. Excluded cardiac, neurosurgery, burns, transplant, minor surgery not requiring GA/RA.

- Data analyzed: Preoperative anemia according to WHO classification, RCRI score, ASA score, type of anaesthesia, priority and severity of surgery (ESA classification), perioperative blood transfusion, post operative baseline and highest serum troponin T within 30 days.

- Outcomes: 30-day mortality obtained from eHINTS which is synced with the National Death Registry and 30-day perioperative myocardial infarction (PMI) defined as a baseline increase of $>=20 \%$ and $>=30 \mathrm{ng} / \mathrm{ml}$ in serum troponin $T$

- Sensitivity analysis: Effect of perioperative blood transfusion

\section{Results}

\section{Overall results}

- Prevalence of preoperative anemia in cohort was $\underline{39.5 \%}$, of which $21.4 \%$ was mild, $17.5 \%$ moderate and $0.6 \%$ severe.

- Incidence of 30-day mortality was $1.4 \%$

- Incidence of 30-day perioperative myocardial infarction as defined as a baseline increase of $>=20 \%$ and $>=30 \mathrm{ng} / \mathrm{ml}$ in troponin $\mathrm{T}$ was $1.8 \%$.

Variables that independently increase 30-day mortality and PMI

- Higher ASA score $(\geq 3)$

- Age $\geq 76$ years

- Presence of anemia

- Emergency surgery (c.f. elective surgery)

- Repeat surgery during admission

- $\mathrm{RCRI}$ scores $\geq 1$

\section{Perioperative transfusion}

- Significantly associated with both outcomes

- 30-day mortality: 1 unit (aOR 1.59, $1.07-2.37$, $\mathrm{p}=0.02$ ); 2 or more units (aOR 2.84, $1.74-4.63$, $p<0.001)$

- 30-day PMI: 1 unit (aOR 2.25, 1.66 - 3.06, $\mathrm{p}<0.001$ ); 2 or more units (aOR 1.67, $1.01-2.77$, $p=0.046)$

\section{Preoperative anaemia}

- Greater anaemia severity associated with worse 30-day outcomes of mortality and 30-day PMI

- Anaemia is associated with greater incidence of perioperative blood transfusion (mild anemia aOR 3.26, $2.75-3.87, p<0.001 ; \mathrm{mod} / \mathrm{severe}$ anemia aOR 12.83, $10.98-14.99, p<0.001$ )

- After adjusting for perioperative transfusion in table 1 , anaemia still has a reduced but independent effect on 30-day outcomes

Table 1. Odds of the anemia severity on 30-day mortality and perioperative myocardial injury (PMI)

\begin{tabular}{c|c|c|c|c} 
& \multicolumn{2}{|c|}{ *aOR of 30-day Mortality } & *aOR of 30-day Myocardial Infarction \\
\hline & & $\begin{array}{c}\text { Adjusted for } \\
\text { transfusion }\end{array}$ & & $\begin{array}{c}\text { Adjusted for } \\
\text { transfusion }\end{array}$ \\
\hline Mild anemia & $\begin{array}{c}1.82(1.61-2.85), \\
p=0.009\end{array}$ & $\begin{array}{c}1.70(1.07-2.64), \\
p=0.025\end{array}$ & $\begin{array}{c}1.52(1.09-2.11), \\
p=0.014\end{array}$ & $\begin{array}{c}1.37(0.98-1.92), \\
p=0.065\end{array}$ \\
\hline $\begin{array}{c}\text { Mod/severe } \\
\text { anemia }\end{array}$ & $\begin{array}{c}2.78(1.87-4.14), \\
\mathrm{p}<0.001\end{array}$ & $\begin{array}{c}2.12(1.39-3.25), \\
\mathrm{p}=0.001\end{array}$ & $\begin{array}{c}1.83(1.35-2.48), \\
\mathrm{p}<0.001\end{array}$ & $\begin{array}{c}1.41(1.01-1.97), \\
\mathrm{p}=0.042\end{array}$
\end{tabular}

* Corrected for ASA status, gender, age, surgery risk category, grade of preoperative kidney disease, repeat surgeries done during admission, RCRI score, ASA score, type of anaesthesia, surgery priority, race

\section{Conclusions}

Pre-operative anaemia in the elderly significantly increased the odds of 30-day in-hospital mortality and perioperative myocardial infarction, and this effect is independent of perioperative blood transfusion. 